\title{
Effect of Four Wave Mixing on AP-DCDM- WDM Fiber Optic System with Different Power per Channel
}

\author{
${ }^{1}$ Farman Ullah, ${ }^{2}$ Aamir Khan, ${ }^{3}$ Nadia N Qadri, ${ }^{4}$ Muhammad MasoodSarfraz \\ 1,2,3,4 Electrical Engineering Department COMSATS Institute of Information Technology, Wah, Pakistan \\ E-mail:farman@ciitwah.edu.pk; engr.aamirkhan@gmail.com;drnadia@ciitwah.edu.pk; mmasood@ciitwah.edu.pk
}

\begin{abstract}
Absolute polar duty cycle division multiplexing over WDM is a multiplexing technique which promises better spectral efficiency. Non linearities in fiber optic communication are major issues, especially the effect of four wave mixing. This paper presents the effect of four wave mixing on $40 \mathrm{Gbps}$ APDCDM over WDM fiber optic systems. The system was tested by simulating the AP-DCDM-WDM design in Optisystem software and MATLAB code. From the results the effect of four wave mixing on the system is presented under different configurations such as input power per channel. Simulation results have shown that AP-DCDM-WDM systems have greater tolerance to dispersion and have better receiver sensitivity than other conventional techniques. The effect of FWM on APDCDM-WDM system is also less than on other conventional techniques on basis of simulation.
\end{abstract}

Index Terms-AP-DCDM, Four Wave Mixing, Channel Spacing, Power per Channel

\section{Introduction}

To use the available bandwidth in optical communication systems different multiplexing techniques are adopted, so that multiple users can access and use the bandwidth efficiently. The most common techniques are frequency division multiplexing, time division multiplexing, optical code division multiplexing, wave length division multiplexing and dense wavelength division multiplexing.

In FDM, users share the bandwidth according to the frequencies allotted to them, therefore, for a given bandwidth the number of users to access the bandwidth and use for communication are limited.

TDM allows users to share bandwidth according to the time slots allotted to them. This is an efficient technique [1]. But the electronic devices required for multiplexing and de-multiplexing channels become bottleneck [2].

WDM allows the users to share bandwidth according to the wave lengths assigned to them. This technique has been used for long and is one the most common techniques for optical communication but it has low spectral efficiency as it uses wide range of wavelengths.

Absolute Polar Duty Cycle Division Multiplexing over wave length division multiplexing technique is another technique which increases the channel count from other conventional existing systems [3].

However, AP-DCDM-WDM system faces the nonlinearities such as self phase modulation, cross phase modulation, four wave mixing etc. as like the other conventional system which affects the spectral efficiency. There is a need to study the effect of non linearities, especially effect of four wave mixing on this technique, to make this technique more efficient and powerful by taking suitable measures against the nonlinearities.

The content of this paper is organized as the $2^{\text {nd }}$ section emphasizes the concept of APDCDM. Section 3 explains the work execution steps and simulation setup for this work. Section 4 gives the results produced in "Section 3" and also comments on results. While the final section then concludes the paper and gives future recommendation.

\section{Absolute Polar Duty Cycle Division Multiplexing (AP-DCDM)}

Day by day, need of large bandwidth for high speed data transfer is growing rapidly. To give enough bandwidth to each user and to accommodate more users sharing bandwidth at one time is one of the major issues.

\section{Overview}

Before optical communication, time division multiplexing was the optimized multiplexing technique for communication systems. After fiber optic cable is being used more widely for high speed data transfer, wavelength division multiplexing does the job [4]. But using absolute polar duty cycle division multiplexing, which is from the family unit of duty cycle division multiplexing over WDM Channels increase the channel count because of using narrow filters [4]. It basically works on the principle of polar signaling and different 
return to zero duty cycles. In other words, it uses bipolar signals with different duty cycles to differentiate between users or channels. Fig. 1 shows the system [5].

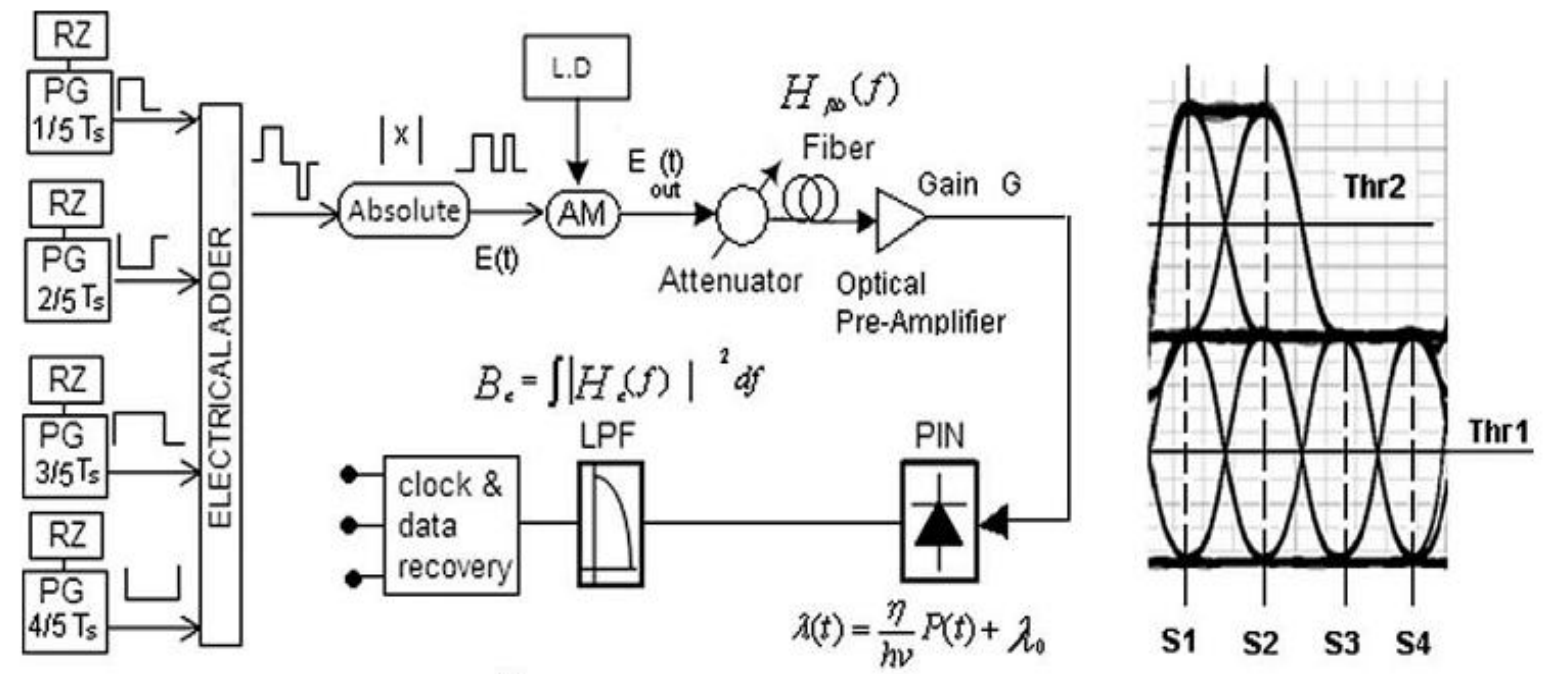

Fig.1: AP-DCDM system for four users [5]

In this technique, the polarity of subsequence users is opposite which results in a unique multilevel pattern at the multiplexer output [5]. Because of this uniqueness it becomes easier at the receiver end to recover the data for each user. AP-DCDM technique can be used with guard bands as well as without guard bands as per requirement [6]. Also this technique is different from other conventional techniques due to the fact that users share the same transmission medium, in same time period with same carrier wavelength but using different duty cycles, which results in uniqueness that is discussed above [6].

\subsection{AP-DCDM Less Prone to Dispersion}

AP-DCDM technique reduces the spectral width and it has small spectral width, due to this fact, has greater spectral efficiency and tolerance towards dispersion [7]. In Fig. 2, the dispersion tolerance is shown for $40 \mathrm{~Gb} / \mathrm{s}$ $50 \%$ duty cycles RZ and 40Gb/s G AP-DCDM [5].

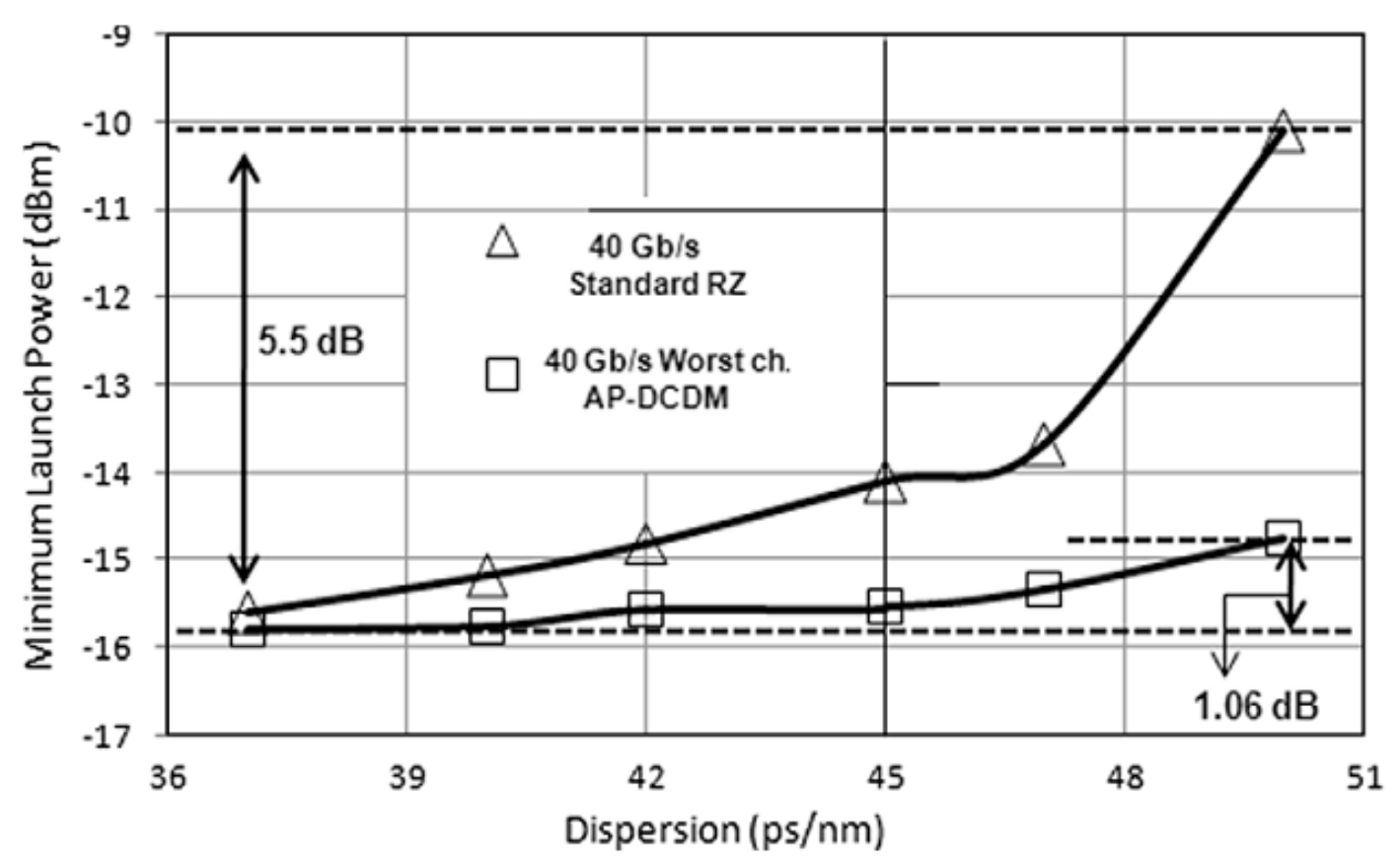

Fig 2:Dispersion tolerance [5] 
In AP-DCDM technique, the uses of narrow filters provide less inter channel coherent cross talk. APDCDM is a new technique which can be implemented over WDM systems to get the optimized system efficiency [8].

\section{Simulation Setup for Effect of Four Wave Mixing}

Analyzing the results from receiver sensitivity and effect of dispersion gave enough understanding of optical communication systems concepts and made the way for studying the effect of four wave mixing on APDCDM-WDM optic fiber systems. Several steps were taken to achieve the final goal. Table 1 gives the values of the parameters which were used for the entire design from start till the final system. The bandwidth of the multiplexer and de-multiplexer was kept $50 \mathrm{GHz}$.

Table 1: Parameter setting for FWM

\begin{tabular}{|c|c|c|c|c|c|c|c|c|c|}
\hline \multicolumn{2}{|c|}{ Main } & Disp... & PMD & Nonl... & Num.. & Gr... & Simu... & Noise & \multirow{2}{*}{$\begin{array}{r}\text { Rand... } \\
\text { Mode }\end{array}$} \\
\hline Disp & \multicolumn{4}{|c|}{ llame } & \multicolumn{3}{|c|}{ Value } & Units & \\
\hline & \multicolumn{4}{|c|}{ User defined reference wa } & \multicolumn{3}{|c|}{$\Gamma$} & & Normal \\
\hline$\Gamma$ & \multicolumn{4}{|c|}{ Reference wavelength } & \multicolumn{3}{|r|}{1550} & $n m$ & Normal \\
\hline$\Gamma$ & \multicolumn{4}{|c|}{ Length } & \multirow{2}{*}{\multicolumn{3}{|c|}{80}} & $k m$ & Normal \\
\hline$\Gamma$ & \multicolumn{4}{|c|}{ Attenuation effect } & & & & & Normal \\
\hline$\Gamma$ & \multicolumn{4}{|c|}{ Attenuation data type } & \multicolumn{3}{|l|}{ Constant } & & Normal \\
\hline$\Gamma$ & \multicolumn{4}{|c|}{ Attenuation } & \multicolumn{3}{|r|}{0.2} & & Normal \\
\hline I & \multicolumn{4}{|c|}{ Attenuation vs. wavelengt } & \multicolumn{2}{|c|}{ Attenuabion. dat } & .] & ] & Normal \\
\hline Mait & & Disp... & PMD & Nonl... & Num ... & Gr... & Simu... & Noise & Rand... \\
\hline Disp & & & ime & & & alue & & Units & Mode \\
\hline & Gr & oup veloc & ty disp & ersion & & $\bar{\sigma}$ & & & Normal \\
\hline & Th: & ir d-order & dispers & ion & & $\sqrt{\sigma}$ & & & Normas \\
\hline 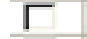 & Dis & sper sion & lata typ & & Constant & & & & Normal \\
\hline$\Gamma$ & Fre & equency c & omain & parame & & & & & Normal \\
\hline & Dis & spersion & & & & & d & $p \mathrm{~s} / \mathrm{nm} / \mathrm{km}$ & Normel \\
\hline$\Gamma$ & Dis & spersion & lope & & & & 0.075 & $P S / n m^{n} 2 / k$ & Normas \\
\hline$\Gamma$ & Be & $\operatorname{ta} 2$ & & & & & -20 & $\rho s^{n} 2 / \mathrm{km}$ & Normal \\
\hline$\Gamma$ & Be: & ta 3 & & & & & 0 & $p s^{\prime} 3 / k m$ & Normel \\
\hline$\Gamma$ & Dis & spersion 1 & lie forn & & Dispersions & wave & ength & & Normal \\
\hline$\Gamma$ & Dis & sper sion 1 & le nam & & Dispersion. & & ... & & Normal \\
\hline Mair & & Disp... & PMD & Nonl.. & Num... & Gr... & Simu... & Noise & Rand... \\
\hline Disp & & & ime & & & alue & & Units & Mode \\
\hline 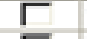 & Sel & If-phase I & vodulat & ion & & $\sqrt{\sigma}$ & & & Normas \\
\hline$\Gamma$ & Eff: & ective are & data t & ype & Constant & & & & Normal \\
\hline$\Gamma$ & Enf & ective are & & & & & 64 & $2 m^{n} 2$ & Normas \\
\hline$\Gamma$ & Ent: & ective are & vs. w. & welen & ErrectiveAra & tat & ... & & Normal \\
\hline$\Gamma$ & $\mathbf{n} 2$ & data type & & & Constant & & & & Normal \\
\hline$\Gamma$ & $\mathbf{n} 2$ & & & & & & $266-021$ & $m^{n} 2 N$ & Normal \\
\hline$\Gamma$ & $n 2$ & vs. wavel & ength & & n2. chat & & $m$ & & Normas \\
\hline$\Gamma$ & Set & If-steepet & ing & & & $\Gamma$ & & & Normal \\
\hline$\Gamma$ & Ful & 11 Raman F & espons & & & $\Gamma$ & & & Nowmal \\
\hline$\Gamma$ & Int: & rapulse R & iman 5 & catt. & & $\Gamma$ & & & Novmes \\
\hline$\Gamma$ & Rar & man self- & hift tin & ve1 & & & 14.2 & 19 & Normas \\
\hline$\Gamma$ & Rar & man self- & hift tin & ne2 & & & 3 & fs & Normas \\
\hline$\Gamma$ & Fra & act. Paman & contrit & rution & & & 0.18 & & Novmal \\
\hline$\Gamma$ & Ort & thogonal & taman $t$ & actor & & & 0.75 & & Normal \\
\hline
\end{tabular}

Initially a design was adopted for WDM system where no external modulator was used. Later on WDM systems with external modulator were experimented and finally the effect of four wave mixing was analyzed on AP-DCDM-WDM System.

\subsection{Simulation Setup for WDM System without External Modulator}

In this design a WDM system was employed without using external modulator. The effect of four wave mixing was studied by analyzing the optical spectrum available in Optisystem and the average power of FWM against power per channel and channel spacing were studied. Line width of the $\mathrm{CW}$ laser was kept zero, so only four wave mixing effect is analyzed. Fig. 3 shows the simulation setup adopted. 


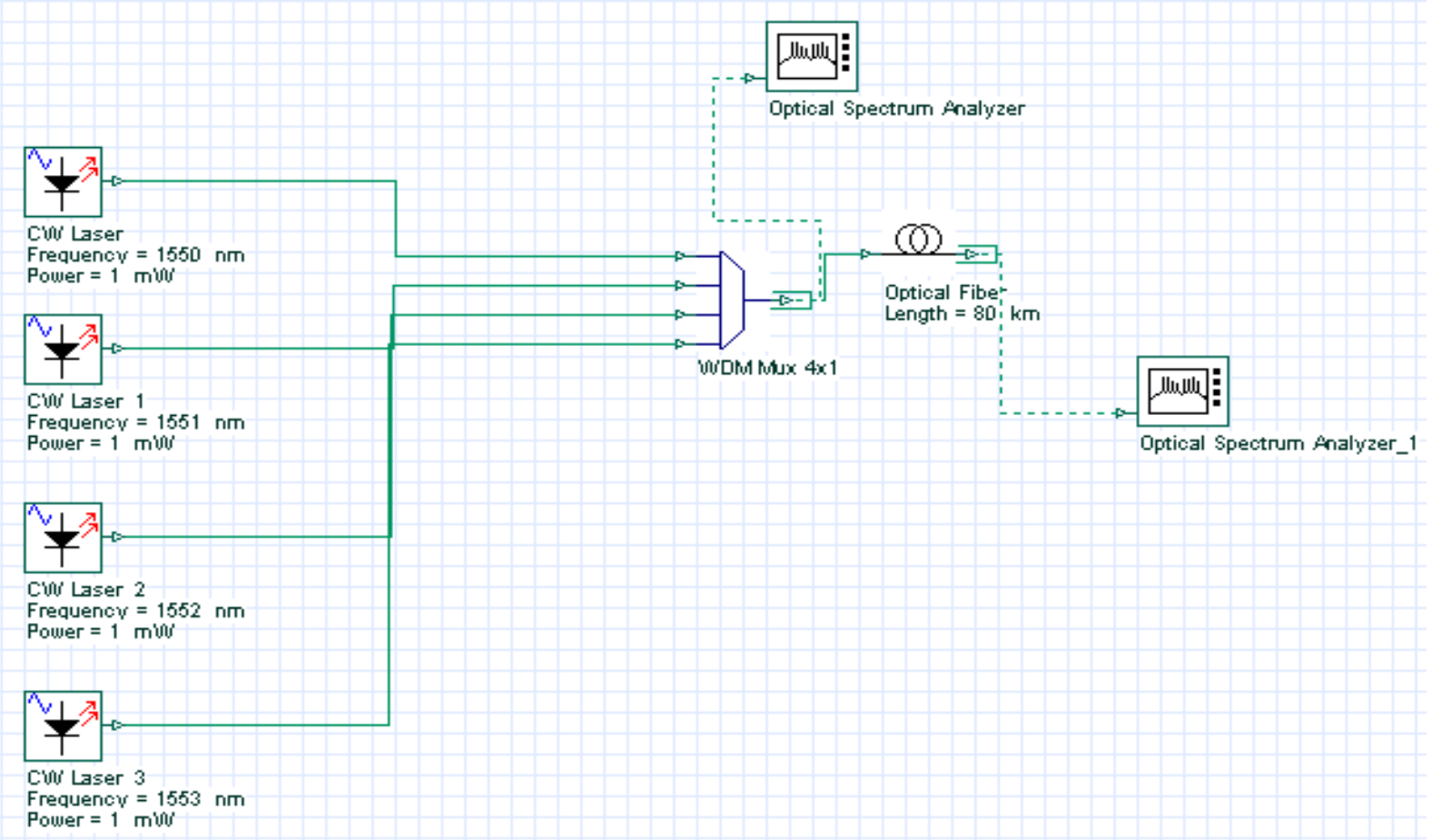

Fig. 3: WDM system without External Modulator

This design was simulated for different channel spacing at $10 \mathrm{Gbps}$ and $40 \mathrm{Gbps}$. Also it was tested for power per channel against average four wave mixing power for $0.4 \mathrm{~nm}$ at $10 \mathrm{Gbps}$ and $40 \mathrm{Gbps}$, and $0.8 \mathrm{~nm}$ channel spacing at $10 \mathrm{Gbps}$ and $40 \mathrm{Gbps}$.

\subsection{Model for RZ and NRZ-WDM Systems with External Modulator}

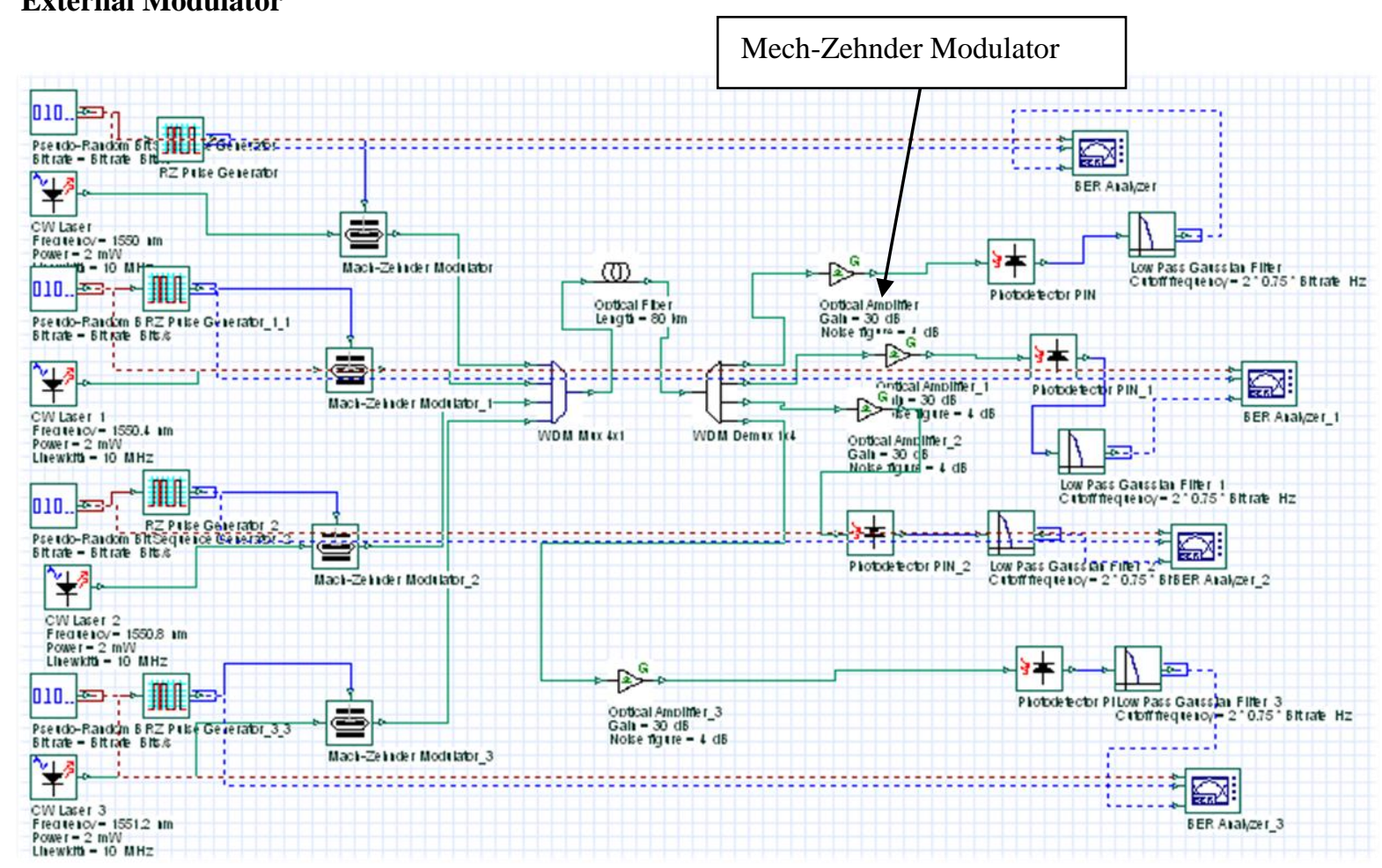

Fig. 4: RZ-WDM System
Mech-Zehnder Modulators were used to produce the modulated signal. Dispersion value is set to zero, so that in calculation of BER only the four wave mixing effect is calculated. The simulation setup for RZ-WDM system operation on 10 Gbpsand $40 \mathrm{Gbps}$ is given in Fig. 4. 
The above system was tested for four channels and was examined for bit rate of $10 \mathrm{Gbps}$ and $40 \mathrm{Gbps}$ for $0.4 \mathrm{~nm}$ and $0.8 \mathrm{~nm}$ channel spacing between each channel. Different power per channel was simulated to see the effect of four wave mixing in the form of BER for each of the system with $0.4 \mathrm{~nm}$ and $0.8 \mathrm{~nm}$ channel spacing and at bit rate of 10 and 40 Gbps. Also this system was tested for different channel spacing operating at $10 \mathrm{Gbps}$ and $40 \mathrm{Gbps}$.

The whole procedure was repeated for 8 channels RZ-WDM System as well. Fig5.shows the setup.

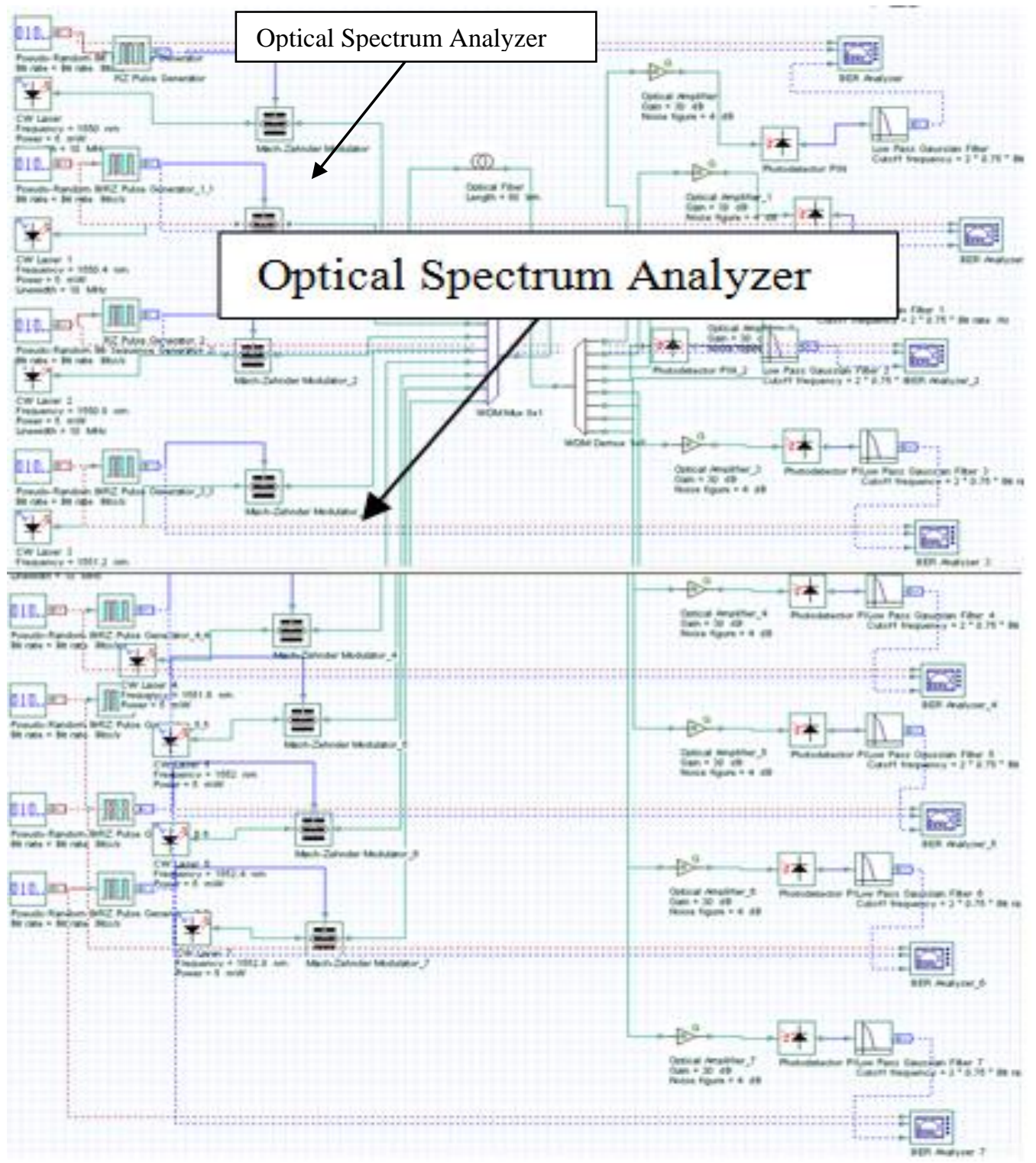

Fig. 5: RZ-WDM 8 Channels System

The design discussed above was then extended for NRZ-WDM system.

\subsection{Simulation setup for AP-DCDM-WDM System}

After finishing the simulation for RZ-WDM system and NRZ-WDM system, and getting the results for the effect of four wave mixing, the final objective of the paper that is, on AP-DCDM-WDM System was simulated. Here Absolute Polar Duty Cycle Division multiplexing is used with WDM to achieve better performance under the effect of four wave mixing yet at high bit rate [9]. In this setup $4 \mathrm{RZ}$ pulse generators are used with the following configuration from first user to the fourth shown in Table 2. 
Table 2: RZ Pulse Generators Configuration

\begin{tabular}{|c|c|c|c|c|}
\hline \multicolumn{5}{|c|}{ Main Simulation } \\
\hline Disp & Ilame & Value & Units & Mode \\
\hline 厂 & Rectangle shape & Exponential & & Normal \\
\hline$\Gamma$ & Amplitude & 1 & a.u. & Normal \\
\hline$\Gamma \mathrm{E}$ & Bias & 0 & a.u. & Normal \\
\hline$\Gamma \mathrm{I}$ & Duty cycle & 0.25 & bit & Normal \\
\hline$\Gamma$ & Position & 0 & bit & Normal \\
\hline$\Gamma$ & Rise time & 0.05 & $b i t$ & Normal \\
\hline$\Gamma$ & Fall time & 0.05 & $b \bar{k}$ & Normal \\
\hline \begin{tabular}{|l|} 
Disp \\
\end{tabular} & llame & Value & Units & Mode \\
\hline$\Gamma$ & Rectangle shape & Exponential & & Normal \\
\hline$\Gamma$ & Amplitude & -1 & a.u. & Normal \\
\hline$\Gamma$ & Bias & 0 & a.u. & Norma! \\
\hline$\Gamma$ & Duty cycle & 0.5 & bit & Normal \\
\hline$\Gamma$ & Position & 0 & bit & Normal \\
\hline$\Gamma$ & Rise time & 0.05 & bit & Normal \\
\hline$\Gamma$ & Fall time & 0.05 & bit & Normal \\
\hline Disp & Ilame & Value & Units & Mode \\
\hline$\Gamma$ & Rectangle shape & Exponential & & Normal \\
\hline$\Gamma$ & Amplitude & 1 & a.d. & Normal \\
\hline$\Gamma$ & Bias & 0 & a.u. & Normal \\
\hline$\Gamma$ & Duty cycle & 0.75 & bit & Normal \\
\hline$\Gamma$ & Position & 0 & bit & Normal \\
\hline$\Gamma$ & Rise time & 0.05 & bit & Normal \\
\hline$\Gamma$ & Fall time & 0.05 & bit & Normal \\
\hline Disp & Ilame & Value & Units & Mode \\
\hline$\Gamma$ & Rectangle shape & Exponential & & Normal \\
\hline$\Gamma$ & Amplitude & -1 & a.u. & Normal \\
\hline$\Gamma$ & Bias & 0 & a.u. & Normal \\
\hline$\Gamma$ & Duty cycle & 1 & $b i$ & Normal \\
\hline$\Gamma$ & Position & 0 & $b i t$ & Normal \\
\hline$\Gamma$ & Rise time & 0.05 & bit & Normal \\
\hline$\Gamma$ & Fall time & 0.05 & $b i t$ & Normal \\
\hline
\end{tabular}

The four RZ Pulse generators with the above mentioned configuration are first combined by an electric adder and passed through an electric absolute circuit to remove the bi polar nature of the signals and then the signal goes into fork which on its output feds one signal to the AM modulate so that the signal is modulated with one external $\mathrm{CW}$ laser using $\mathrm{AM}$ modulator and the other is fed into matlab component [10][11]. The modulated signal is then fed to one channel of WDM multiplexer and this setup is repeated for as many times as required or available number of channels over WDM. The simulation setup for second channel of WDM 4 X 1 multiplexer is shown in Fig 6 .

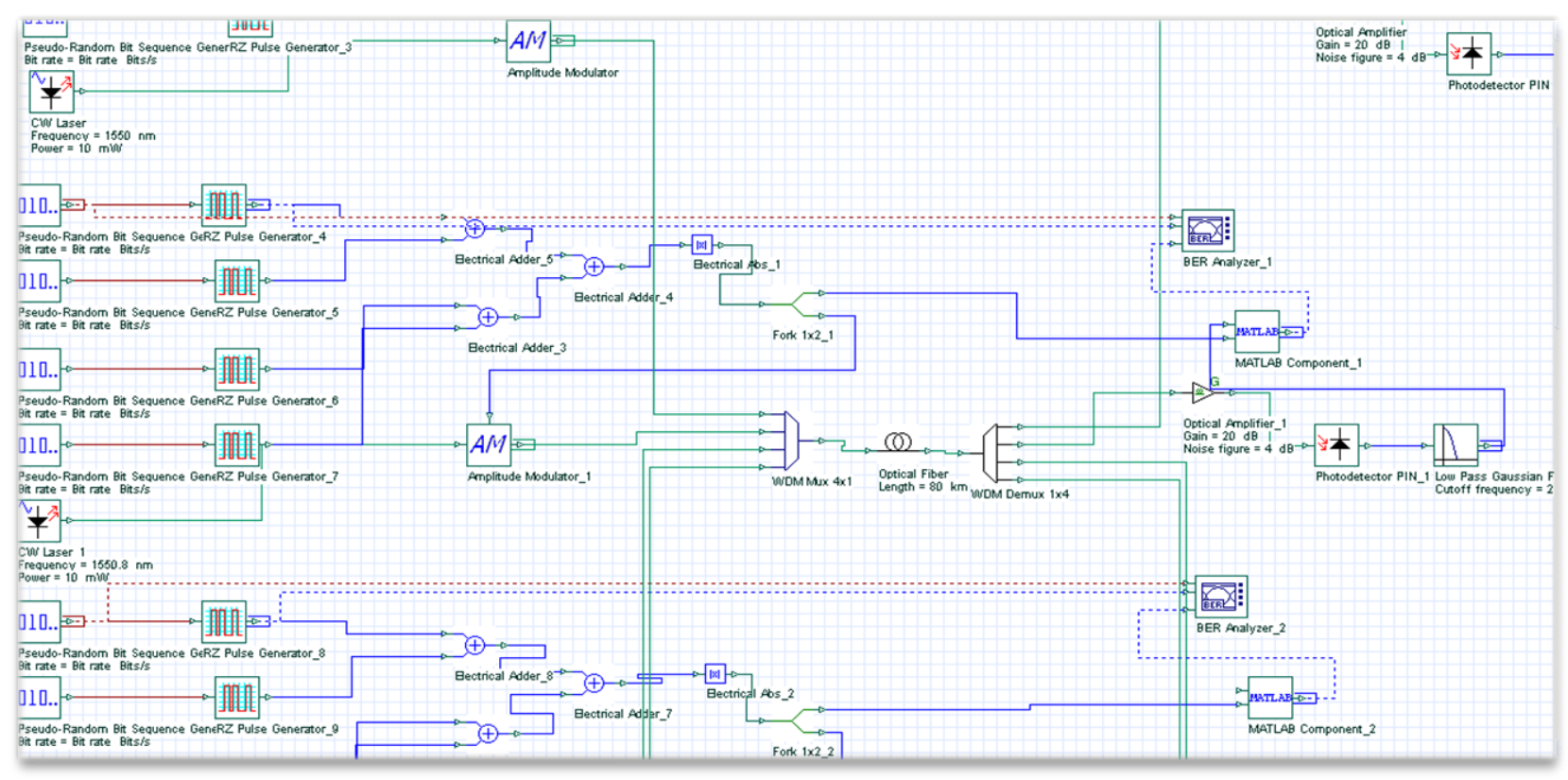

Fig. 6: AP-DCDM-WDM System 
For this design, the dispersion value was kept to zero so only the four wave mixing effect is calculated as the BER of the system. The length of the single mode fiber is $80 \mathrm{~km}$. The effective area of the fiber is $64 \mathrm{um}^{\wedge} 2$. The bit rate is $10 \mathrm{Gbps}$ for each user and the bandwidth of WDM multiplexer is $50 \mathrm{GHz}$. To calculate the BER with zero dispersion, a MATLAB component was used which was attached to a matlab file. Optisystem provides the facility of connecting the layout setup to the matlab files. Matlab file has the appropriate code to do the task and hence the effect of four wave mixing is examined for different power per channel and different channel spacing [12].

\section{Results and Discussion}

\subsection{Effect of Four Wave Mixing on AP-DCDM-} WDM Optic Fiber System
As it was discussed in previous section that APDCDM-WDM the second channel of WDM was examined without guard bands and four users over that channel are studied. In this section the effect of four wave mixing in the AP-DCDM-WDM environment over this second channel of $4 \mathrm{X} 1 \mathrm{WDM}$ multiplexer is examined for four users. The length of the fiber is 80 $\mathrm{km}$ and the effective area of the fiber is $64 \mathrm{um}^{\wedge} 2$. The dispersion is kept zero so while in calculation of BER the effect of four wave mixing is mainly calculated. The sequence length is $1 \mathrm{Mb}$ and the bit rate for each channel of WDM is 40 Gbps while $10 \mathrm{Gbps}$ is the bit rate of each user in the AP-DCDM system. The bandwidth of Multiplexer and Demultiplexer is $50 \mathrm{GHz}$ and Channel spacing are $0.4 \mathrm{~nm}$ and $0.8 \mathrm{~nm}$. Initially the AP-DCDM was designed and simulated. The eye diagram of this system is shown in Fig.7 and Fig. 8 shows the eye diagram of the AP-DCDM system with guard band.

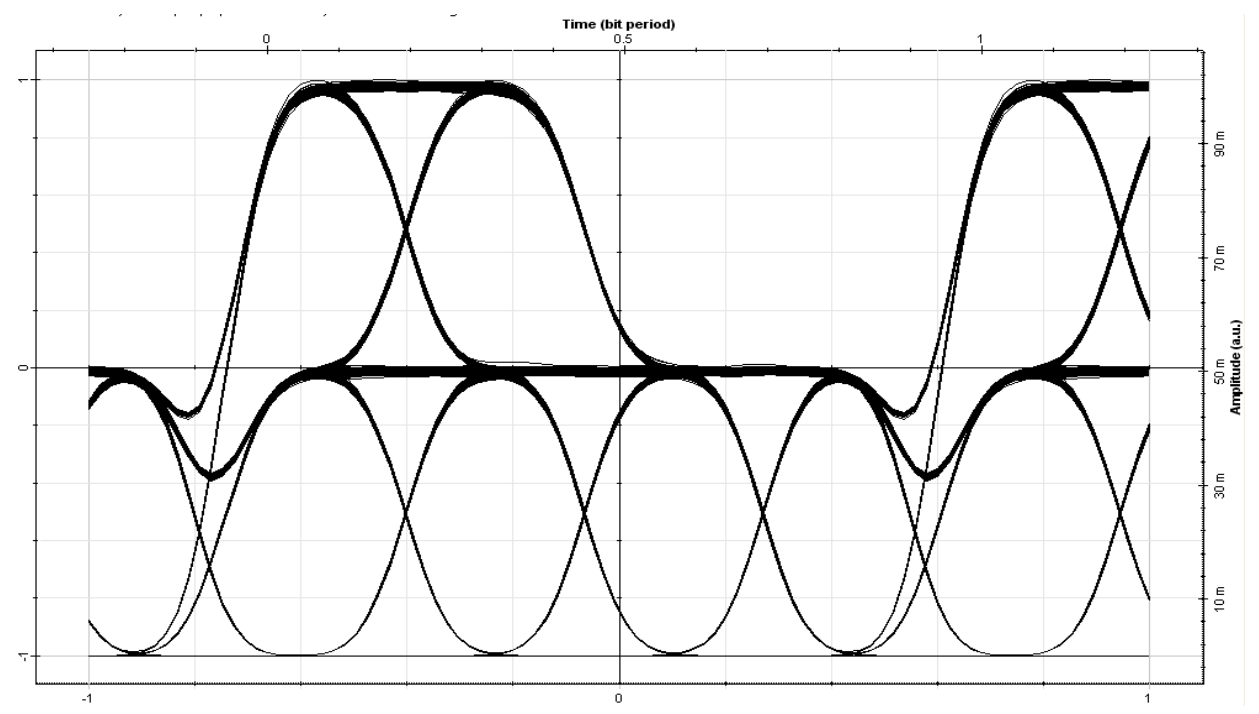

Fig.7: Eye Diagram of AP-DCDM System without Guard Band

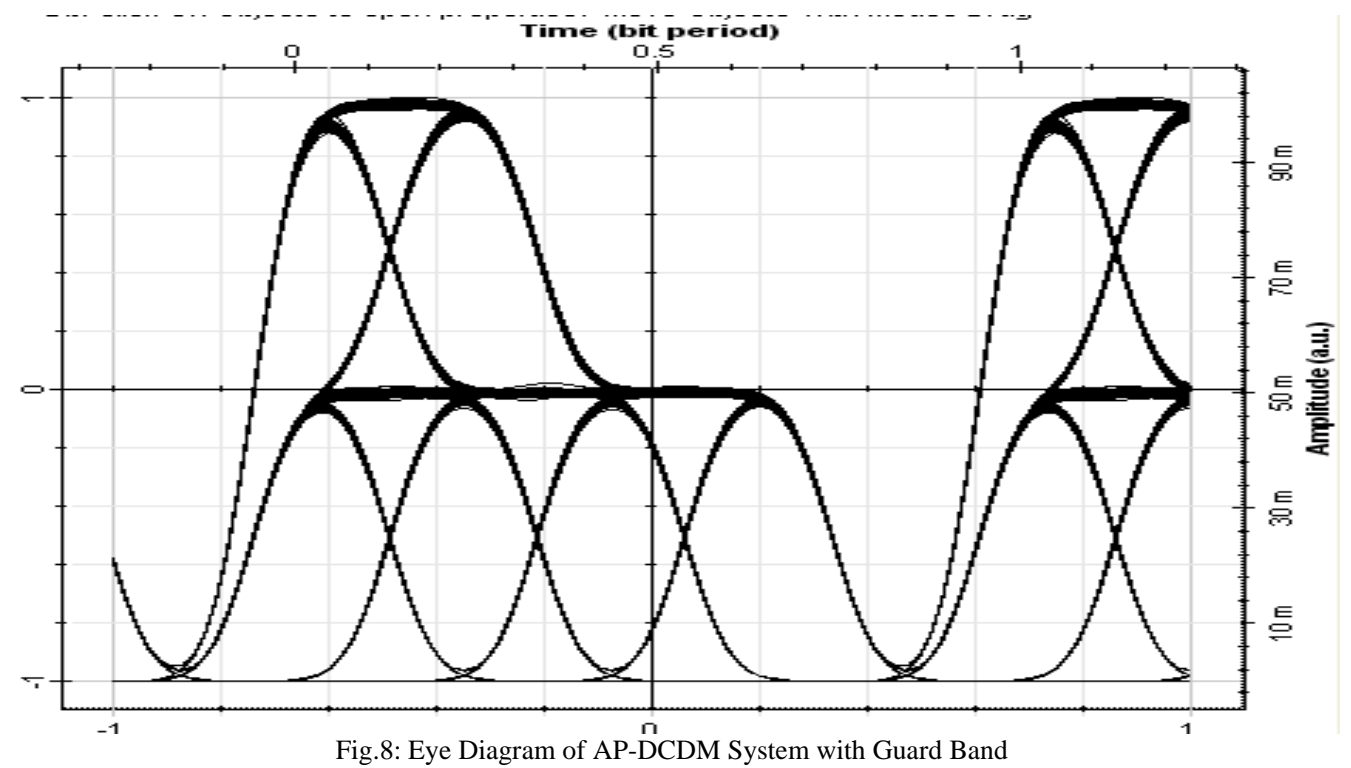


The AP-DCDM-WDM system without guard band is the focus of this paper and simulation under various conditions was performed on this system and the results discussed in this section are related to the AP-DCDMWDM system without guard band.

\subsection{Effect of Power per Channel with $0.4 \mathrm{~nm}$ Channel Spacing}

The AP-DCDM-WDM system was first simulated with $0.4 \mathrm{~nm}$ channel spacing between the channels of WDM. The system was first tested with input power per channel of $1 \mathrm{~mW}$. For which the Fig9 shows the eye diagram.

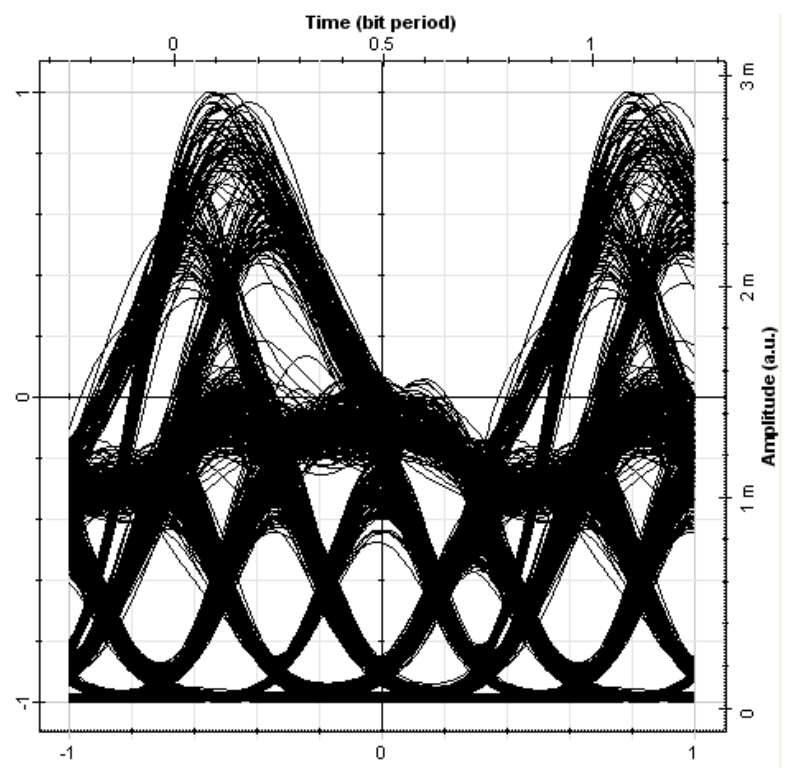

Fig.9: Eye Diagram for input Power per Channel of $1 \mathrm{~mW}$

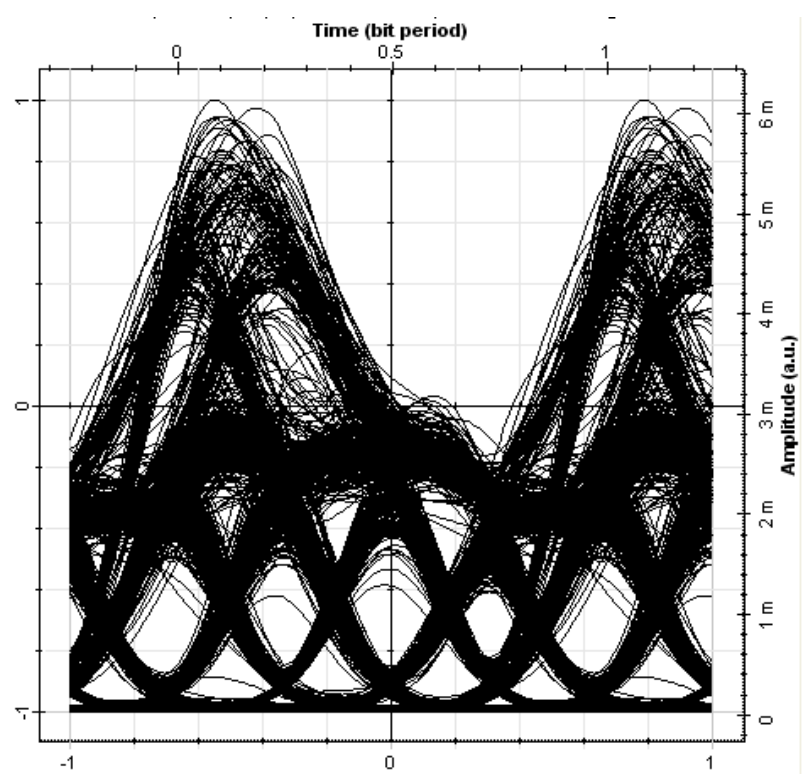

Fig. 10: Eye Diagram for input Power per Channel of $2 \mathrm{~mW}$

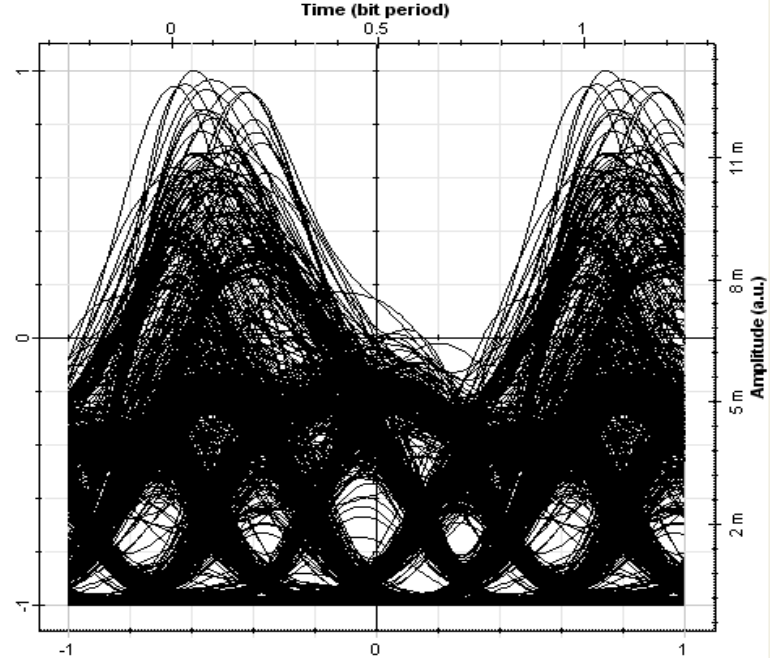

Fig. 11: Eye Diagram for input Power per channel of $4 \mathrm{~mW}$

Then the system was tested for input power of $2 \mathrm{~mW}$, $4 \mathrm{~mW}$ and $6 \mathrm{~mW}$. The results in of these changes to input power per channel are shown in Fig. 10 Fig. 11 and Fig. 12 in the form of eye diagrams respectively. Observing the eye diagrams for each change in input power per channel, it can be stated that as the input power per channel increases the effect of four wave mixing on the system increases.

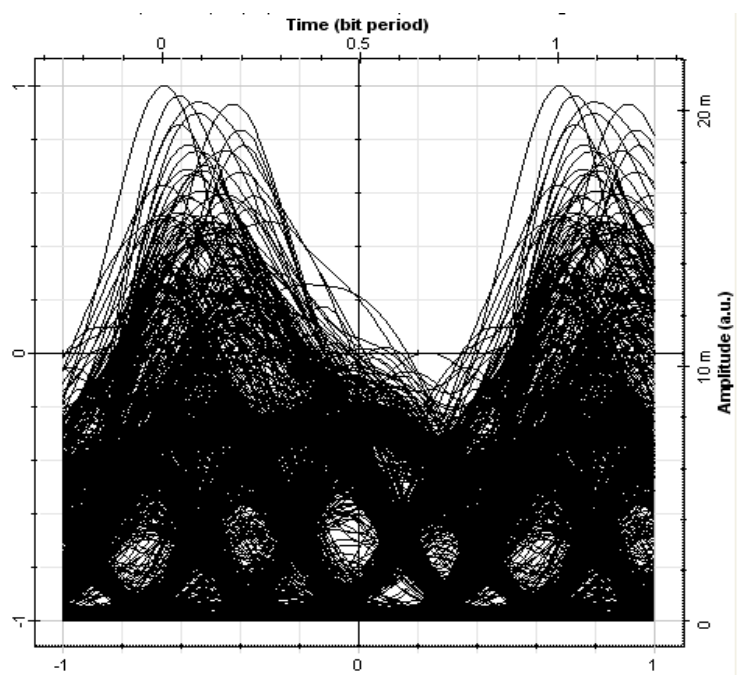

Fig. 12: Eye Diagram for input Power per Channel of $6 \mathrm{~mW}$

\subsection{Effect of Power per Channel with $0.8 \mathrm{~nm}$ Channel Spacing}

When the input power per channel was increased the effect of FWM was seen. The system was tested for input power per channel of $2 \mathrm{~mW}$. Fig 12 shows the eye diagram for the system at input power per channel of 2 $\mathrm{mW}$. It is distorted, which indicates that the effect of four wave mixing is encountered by the system and causing the BER to get worst. 


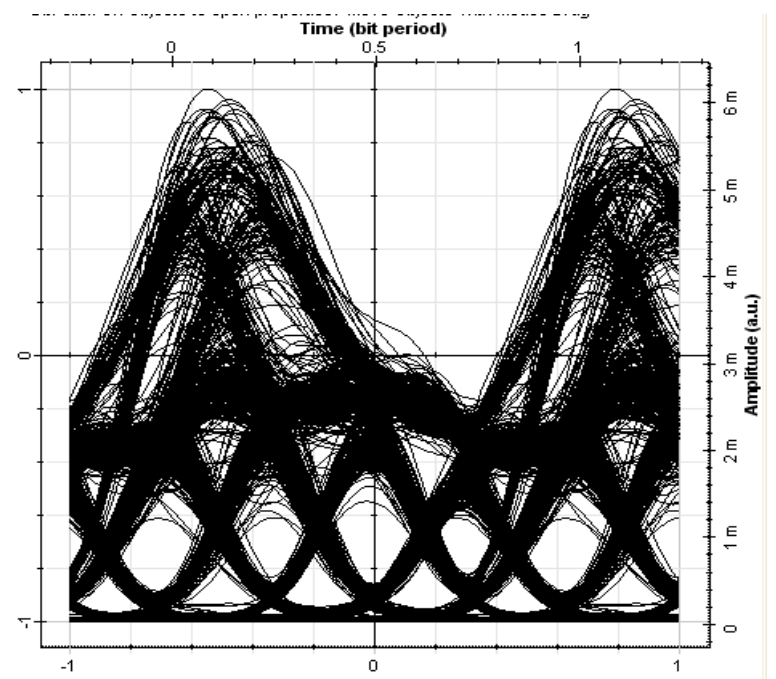

Fig. 13: Eye Diagram at input Power per Channel of $2 \mathrm{~mW}$

The input power per channel was then increased to 4 $\mathrm{mW}$ and simulated the design. From the eye diagram shown in Fig. 13, the effect of four wave mixing on the system is obvious as the eye diagram is distorted more and the effect gets severed when the input power per channel is increased to $6 \mathrm{~mW}$ and $8 \mathrm{~mW}$ subsequently, for which the eye diagrams are shown in Fig 14 and 15 respectively.

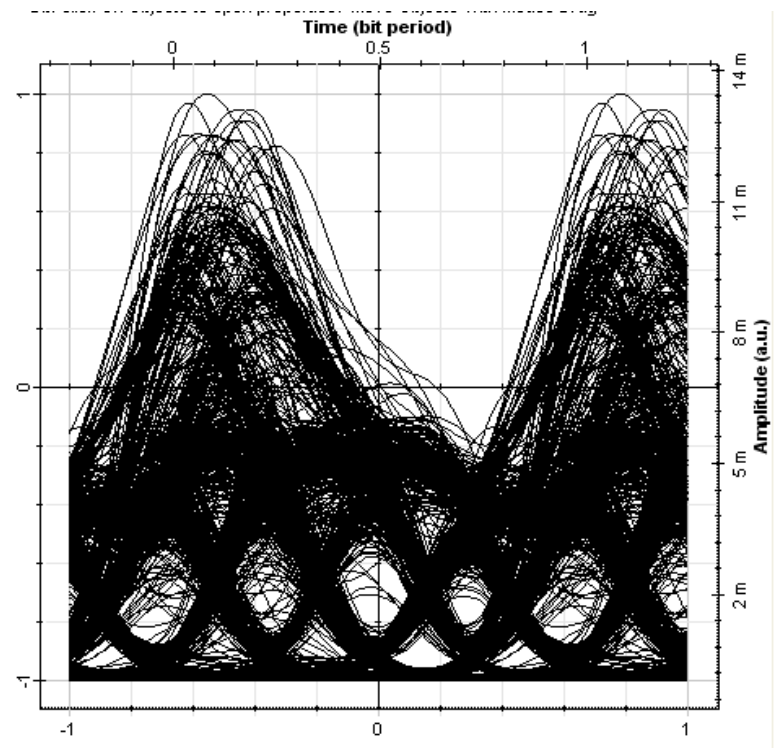

Fig.14: Eye Diagram at input Power per channel of $4 \mathrm{~mW}$

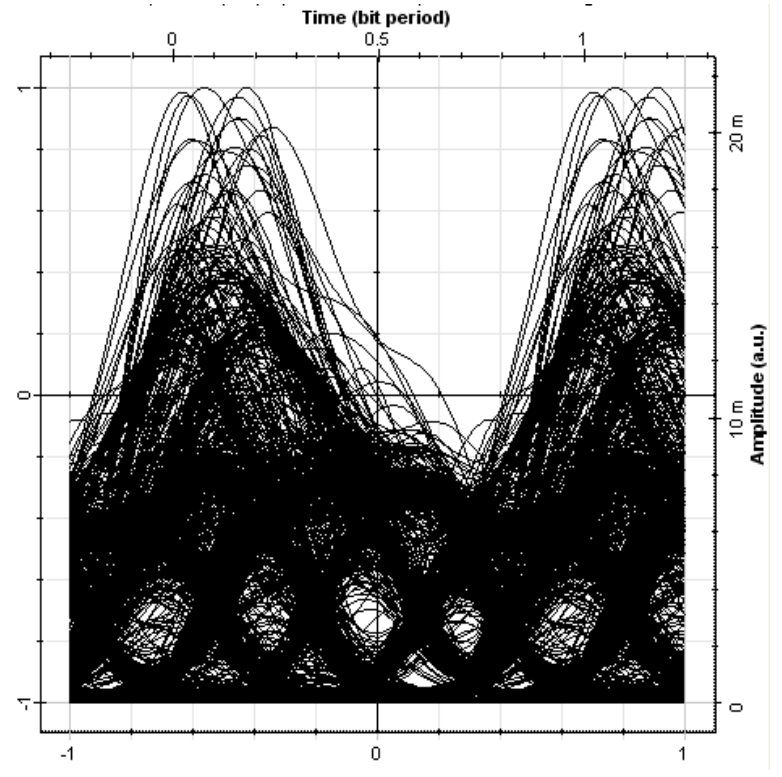

Fig.15: Eye Diagram at input Power per Channel of $6 \mathrm{~mW}$

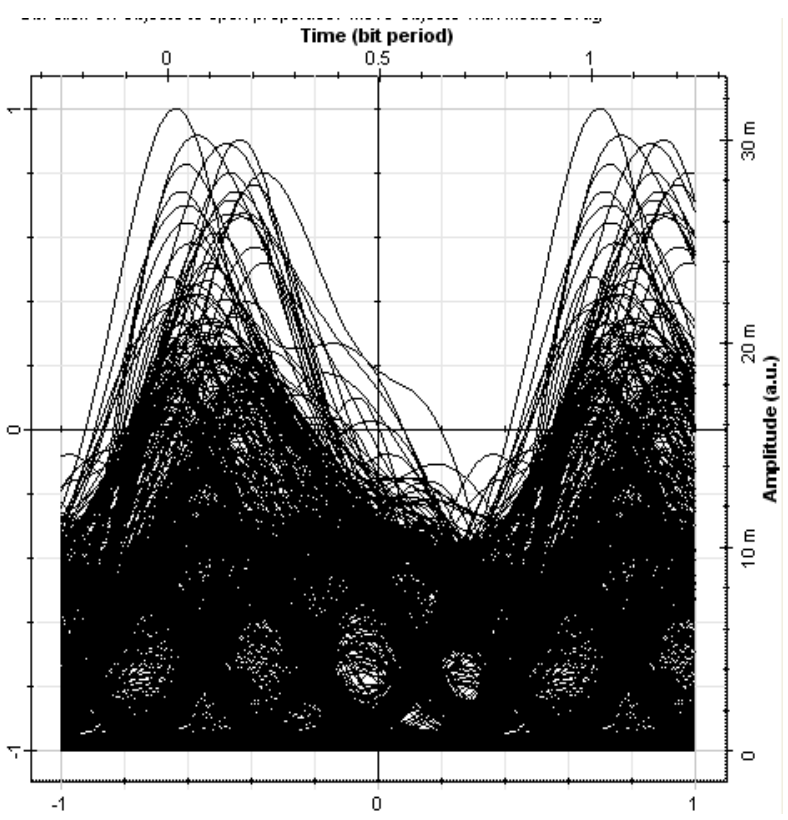

Fig.16: Eye Diagram at input Power per Channel of $8 \mathrm{~mW}$

For the above discussed values of the input power per channel the overall system response is depicted in Fig. 17. 


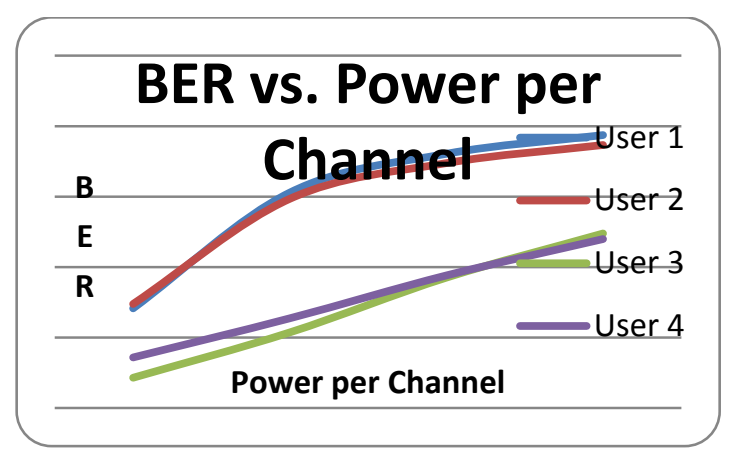

Fig. 17: BER vs. Power per Channel

Obtaining the results as shown in the above figure and observing the eye diagrams for different input power per channel the fact is quite obvious to state that as the input power per channel increases the effect of four wave mixing on the system increases. To transmit the signal over long distance input power is needed but the fact just observed is that by increasing the power the effect of four wave mixing on system increases and transmitted information may be lost due to corrupted data. Though, there is clear restriction on input power per channel, but techniques such as unequal channel spacing and in presence of dispersion the effect of four wave mixing even when the input power is increased can be reduced.

\section{Conclusion and Recommendation}

In this paper, 40 Gbps AP-DCDM over 4 channels WDM system was analyzed for performance under the effect of four wave mixing which is the main non linearity in optical fiber communication system.

It is obvious from results that as the input power per channel are increased, the effect of four wave mixing also increased. For longer distance, the input power per channel is required for signal strength. For this purpose the unequal channel spacing technique could be used to reduce the effect of four wave mixing.

Moreover, results indicated that when the channel spacing is increased, the effect of four wave mixing is noticed to be decreased. With narrow channel spacing the effect of four wave mixing is more severe and signal gets distorted.

Furthermore, it is evident from results when the number of channels increases, the effect of four wave mixing increases.

Comparing the results of AP-DCDM-WDM system, with RZ-WDM and NRZ-WDM system, it would clearly indicate that AP-DCDM-WDM has more spectral efficiency and is less prone to effect of four wave mixing, while on the other hand NRZ-WDM system is better in performance than RZ-WDM system where the criteria of performance is, receiver sensitivity, effect of dispersion and effect of four wave mixing.
The AP-DCDM-WDM fiber optic system elaborated in this paperand the effect of four wave mixing on it was done when the AP-DCDM system was used without guard band. So, it is recommended to study the effect of four wave mixing on the AP-DCDM-WDM fiber optic system with guard band.

Also in quest of making the spectral efficiency better, techniques for reducing the effect of four wave mixing can be proposed such as in the presence of dispersion, the effect of power per channel can be tested.

\section{References}

[1] G.E.Kaiser, Optical Fiber Communications, 3rd edition, McGraw Hill, New York.

[2] Harry J. R. Dutton, Understanding Optical Communication, IBM Corporation, 1998.

[3] RazaliNgah, Optical Time Division Multiplexing For Optical Communication System, University Technology Malaysia, 2008.

[4] Amin Malekmohammadi, Ahmad FauziAbas, MohamadKhazani Abdullah, GhafourAmouzadMahdiraji, MakhfudzahMokhtar, MohdFadlee A. Rasid, Absolute Polar Duty Cycle Division Multiplexing over wavelength division multiplexing system, 2009.

[5] Amin Malekmohammadi, Ahmad FauziAbas, MohamadKhazani Abdullah, GhafourAmouzadMahdiraji, MakhfudzahMokhtar, MohdFadlee A. Rasid, Realization of high capacity transmission in fiber optic communication systems using AP-DCDM technique, 2009.

[6] Malekmohammadi, Amin (2009) Absolute Polar Duty Cycle Division Multiplexing for High-Speed Fiber Optic Communication System. PhD paper, Universiti Putra Malaysia.

[7] Govind P. Agrawal, Non Linear Fiber Optics, third edition, Academic Press, 2001.

[8] M.T. Al-Qdah, H.A. Abdul-Rashid, K. Dimyati, B.M. Ali and M. Khazani, Effect of optical beat interference in SCM/WDM optical networks in presence of FWM, KMITL Sci. Tech. J. Vol. 5 No. 3 Jul.-Dec. 2005.

[9] S. P. Singh and N.Singh, Non linear effects in optical fibres: Origin, Management and Applications, Progress In Electromagnetics Research, PIER 73, 249-275, 2007.

[10] Tan Saw Chin, F.M. Abbou and Ewe Hong Tat, Impact of Four Wave Mixing (FWM) in Routing and Wavelength Assignment, American Journal of Applied Sciences 5 (8): 1059-1063, 2008.

[11] Malekmohammadi, Amin (2009) Absolute Polar Duty Cycle Division Multiplexing for High-Speed Fiber Optic Communication System. PhD paper, Universiti Putra Malaysia.

[12] Ezra Ip and Joseph M.Kahn, Fellow, IEEE Power Spectra of Return-to-Zero Optical Signals JOURNAL OF LIGHTWAVE TECHNOLOGY, VOL.24, NO.3, MARCH 2006. 


\section{Authors' Profiles}

Farman Ullah: Lecturer in the department of Electrical Engineering in COMSATS Institute of Information Technology Wah, Pakistan, major in Mobile and Satellite Communications.

Aamir Khan: Lecturer in the department of Electrical Engineering in COMSATS Institute of Information Technology Wah, Pakistan, major in Multimedia Processing, Networking and Optical Comm.

Nadia N Qadri: Associate Professor in the department of Electrical Engineering in COMSATS Institute of Information Technology, Wah, Pakistan major in Mobile Ad hoc Networks, Wireless Networks, Cognitive Radio

M. MasoodSarfraz: Lecturer in the department of Electrical Engineering in COMSATS Institute of Information Technology Wah, Pakistan, major in Electronics and Communication. 